\title{
ANALYSIS OF ULTRASHORT LASER PULSE INTERACTIONS WITH METAL FILMS USING A TWO-TEMPERATURE MODEL
}

\author{
Ewa Majchrzak, Jolanta Dziatkiewicz \\ Institute of Computational Mechanics and Engineering \\ Silesian University of Technology, Gliwice, Poland \\ ewa.majchrzak@polsl.pl,jolanta.dziatkiewicz@polsl.pl
}

\begin{abstract}
In the paper the problem of thin metal film subjected to the action of the high laser fluence and the ultrashort pulse width is considered. The mathematical model consists of the equations describing the electrons and phonons temperatures and the relationships between the heat fluxes and temperature gradients of electrons and phonons. The problem is solved using the explicit scheme of the finite difference method with staggered grid. In the final part the results of computations and conclusions are presented.
\end{abstract}

Keywords: microscale heat transfer, two-temperature model, finite difference method

\section{Introduction}

To describe the heat transfer process in thin metal film subjected to the ultrashort laser pulse action the two-temperature model is used. A key issue in the application of this model is the proper description of temperature dependent thermophysical properties of the material. Special attention should be given to the method for determining the thermal conductivity and volumetric heat capacity of electrons, as well as the coupling factor. This paper presents the dependencies describing the above-mentioned parameters which should be used in the case of high laser fluence and the ultrashort pulse. The problem discussed is solved using the explicit finite difference method. In the final part of the paper the results of the computations are shown.

\section{Governing equations}

Two-temperature model describing the temporal and spatial evolution of the lattice and electrons temperatures $\left(T_{l}\right.$ and $\left.T_{e}\right)$ in the irradiated metal is of the form $[1,2](1 \mathrm{D}$ problem $)$

$$
C_{e}\left(T_{e}\right) \frac{\partial T_{e}(x, t)}{\partial t}=-\frac{\partial q_{e}(x, t)}{\partial x}-G\left(T_{e}\right)\left[T_{e}(x, t)-T_{l}(x, t)\right]+Q(x, t)
$$


and

$$
C_{l}\left(T_{l}\right) \frac{\partial T_{l}(x, t)}{\partial t}=-\frac{\partial q_{l}(x, t)}{\partial x}+G\left(T_{e}\right)\left[T_{e}(x, t)-T_{l}(x, t)\right]
$$

where $T_{e}(x, t), T_{l}(x, t)$ are the temperatures of electrons and lattice, respectively, $C_{e}\left(T_{e}\right), C_{l}\left(T_{l}\right)$ are the volumetric specific heats, $G\left(T_{e}\right)$ is the electron-phonon coupling factor which characterizes the energy exchange between electrons and phonons, $Q(x, t)$ is the source function associated with the irradiation.

Instead of classical Fourier law the following formulas are introduced:

$$
q_{e}(x, t)+\tau_{e} \frac{\partial q_{e}(x, t)}{\partial t}=-\lambda_{e}\left(T_{e}, T_{l}\right) \frac{\partial T_{e}(x, t)}{\partial x}
$$

and

$$
q_{l}(x, t)+\tau_{l} \frac{\partial q_{l}(x, t)}{\partial t}=-\lambda_{l}\left(T_{l}\right) \frac{\partial T_{l}(x, t)}{\partial x}
$$

where $\lambda_{e}\left(T_{e}, T_{l}\right), \lambda_{l}\left(T_{l}\right)$ are the thermal conductivities of electrons and lattice, respectively, $\tau_{e}$ is the relaxation time of free electrons in metals, $\tau_{l}$ is the relaxation time in phonon collisions.

The laser irradiation is described by a source term introduced in equation (1)

$$
Q(x, t)=\sqrt{\frac{\beta}{\pi}} \frac{1-R}{t_{p} \delta} I_{0} \exp \left[-\frac{x}{\delta}-\beta \frac{\left(t-t_{p}\right)^{2}}{t_{p}^{2}}\right]
$$

where $I_{0}$ is the laser intensity, $t_{p}$ is the characteristic time of laser pulse, $\delta$ is the optical penetration depth, $R$ is the reflectivity of the irradiated surface and $\beta=4 \ln 2[1]$.

The heat losses from front and back surface could be neglected, therefore the boundary conditions are as follows:

$$
q_{e}(0, t)=q_{e}(L, t)=q_{l}(0, t)=q_{l}(L, t)=0
$$

The initial condition was proposed as

$$
T_{e}(x, 0)=T_{l}(x, 0)=T_{p}
$$

\section{Thermophysical parameters}

A very important problem is the appropriate estimation of temperature-dependent thermophysical parameters appearing in the above presented model. Usually, the values of the volumetric specific heat $C_{l}\left(T_{l}\right)$ and thermal conductivity $\lambda_{l}\left(T_{l}\right)$ 
of the lattice are assumed as the constants. For low laser intensity the following relationships describing the electrons thermal capacity and volumetric specific heat are widely used [1-4]:

and

$$
\lambda_{e}\left(T_{e}, T_{l}\right)=\lambda_{0} \frac{T_{e}}{T_{l}}
$$

$$
C_{e}\left(T_{e}\right)=A T_{e}
$$

where $\lambda_{0}$ and $A$ are the material constants, and $[3,5]$

$$
A=\frac{\pi^{2} N k_{B}}{2 T_{F}}
$$

while $N$ is the density of electrons, $k_{B}$ is the Boltzmann constant and $T_{F}$ is the Fermi temperature.

In this case also the remaining parameters, meaning coupling factor $G$ and relaxation times $\tau_{e}, \tau_{l}$, usually are assumed to be the constant ones.

For high laser intensity, these formulas should not be used $[3,6-8]$. For volumetric specific heat of electrons it is assumed that the dependence (9) is valid for the temperatures $T_{e}$ below the Fermi temperature $T_{F}$ divided by $\pi^{2}$, while for the temperatures $T_{e}$ above the Fermi temperature the electrons volumetric specific heat is constant and equal to $3 N k_{B} / 2$ [9]. For temperatures $T_{e}$ in the range $\left[T_{F} / \pi^{2}, T_{F}\right]$ the linear temperature dependence can be accepted and then

$$
C_{e}\left(T_{e}\right)=\left\{\begin{array}{lc}
A T_{e}, & T_{e}<T_{F} / \pi^{2} \\
A T_{F} / \pi^{2}+\frac{3 N k_{B} / 2-A T_{F} / \pi^{2}}{T_{F}-T_{F} / \pi^{2}}\left(T_{e}-T_{F} / \pi^{2}\right), & T_{F} / \pi^{2} \leq T_{e}<T_{F} \\
3 N k_{B} / 2, & T_{e}>T_{F}
\end{array}\right.
$$

It should be pointed out that in paper [6] the interval $\left[T_{F} / \pi^{2}, T_{F}\right]$ is divided into two sub-intervals $\left[T_{F} / \pi^{2}, 3 T_{F} / \pi^{2}\right],\left[3 T_{F} / \pi^{2}, T_{F}\right]$ and for $T_{e}=3 T_{F} / \pi^{2}$ it is assumed that $C_{e}\left(T_{e}\right)=N k_{B}$. Next, the linear temperature dependences in the sub-intervals are proposed, namely

$$
C_{e}\left(T_{e}\right)= \begin{cases}A T_{e}, & T_{e}<T_{F} / \pi^{2} \\ A T_{F} / \pi^{2}+\frac{N k_{B}-A T_{F} / \pi^{2}}{2 T_{F} / \pi^{2}}\left(T_{e}-T_{F} / \pi^{2}\right), & T_{F} / \pi^{2} \leq T_{e}<3 T_{F} / \pi^{2} \\ N k_{B}+\frac{N k_{B} / 2}{T_{F}-3 T_{F} / \pi^{2}}\left(T_{e}-3 T_{F} / \pi^{2}\right), & 3 T_{F} / \pi^{2} \leq T_{e}<T_{F} \\ 3 N k_{B} / 2, & T_{e}>T_{F}\end{cases}
$$


In Figure 1 the courses of functions (11) and (12) for gold are shown.

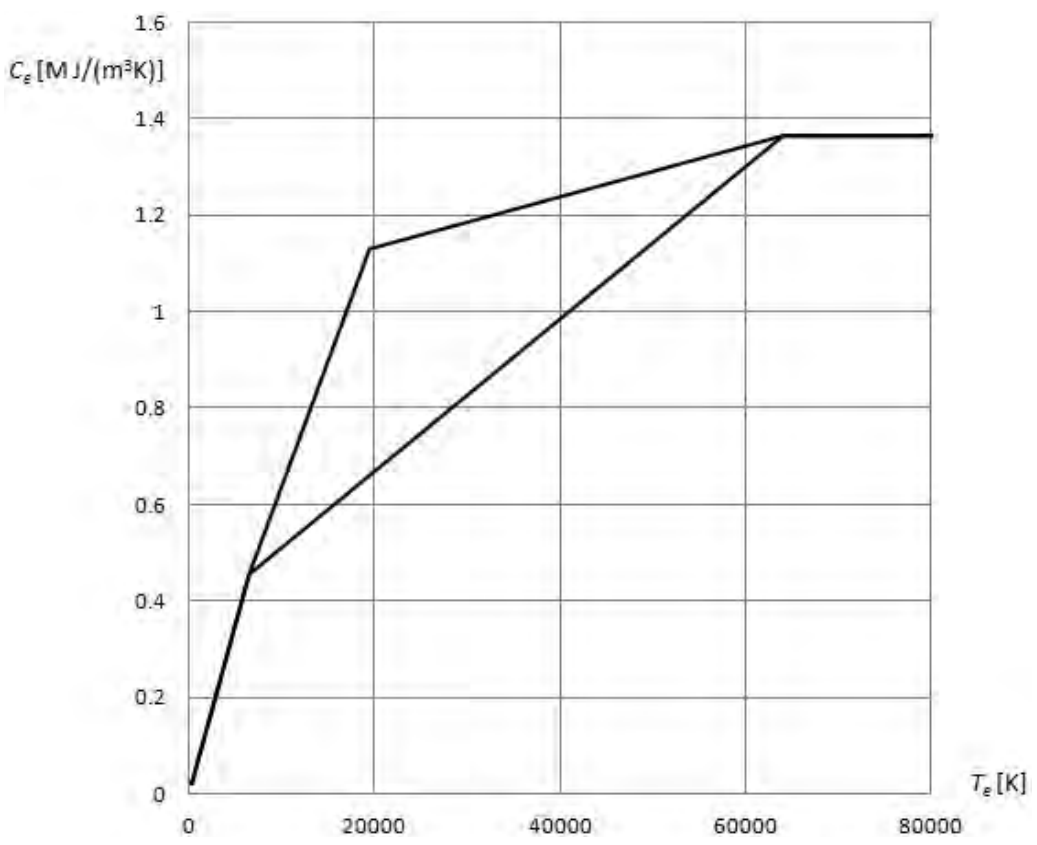

Fig. 1. Function $C_{e}\left(T_{e}\right)$

In the case of electrons thermal conductivity the following dependence is used [10]

$$
\lambda_{e}\left(T_{e}, T_{l}\right)=\chi \frac{\left[\left(T_{e} / T_{F}\right)^{2}+0.16\right]^{5 / 4}\left[\left(T_{e} / T_{F}\right)^{2}+0.44\right]\left(T_{e} / T_{F}\right)}{\left[\left(T_{e} / T_{F}\right)^{2}+0.092\right]^{1 / 2}\left[\left(T_{e} / T_{F}\right)^{2}+\eta\left(T_{l} / T_{F}\right)\right]}
$$

while for the coupling factor

$$
G\left(T_{e}, T_{l}\right)=G_{r t}\left[\frac{A_{e}}{B_{l}}\left(T_{e}+T_{l}\right)+1\right]
$$

where $\chi, \eta, A_{e}, B_{l}$ are the constants and $G_{r t}$ is the coupling factor at room temperature [10].

In Figure 2 the course of electrons thermal conductivity for $300 \leq T_{e} \leq 24000 \mathrm{~K}$ (gold) under the assumption that $T_{l}=300 \mathrm{~K}$ is shown. 


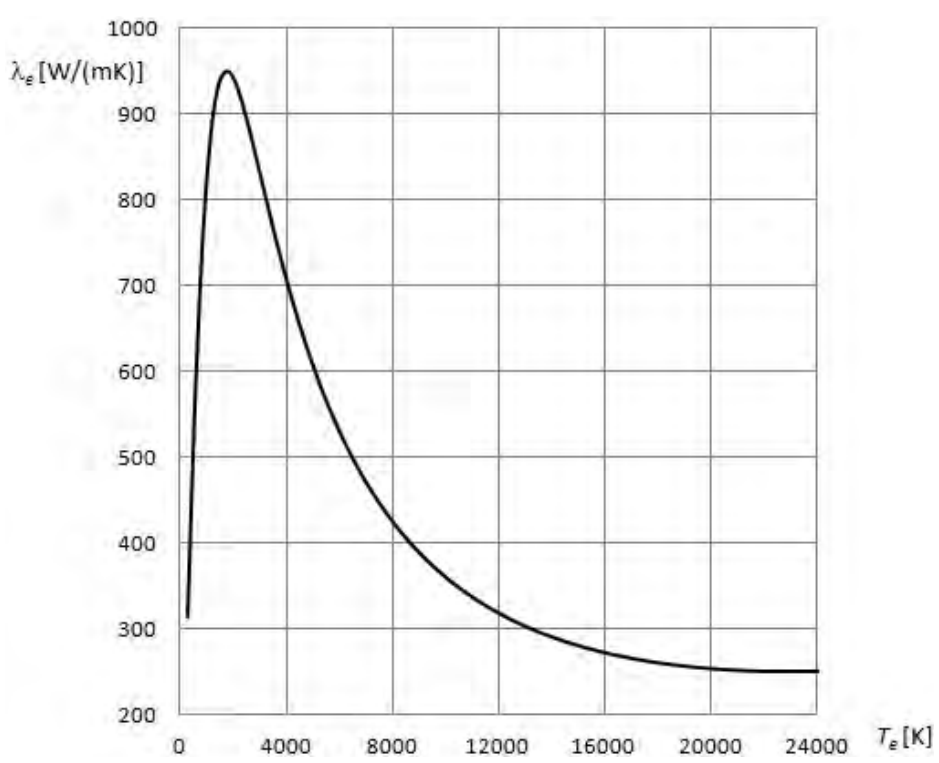

Fig. 2. Electrons thermal conductivity $\left(T_{l}=300 \mathrm{~K}\right)$

\section{Method of solution}

To solve the problem formulated the algorithm based on the finite difference method is used [2]. A staggered grid is introduced in which the temperature nodes $i=0,2,4, \ldots, N$ and the heat fluxes nodes $j=1,3, \ldots, N-1$ are distinguished. Let us denote $T_{i}^{f}=T(i h, f \Delta t)$ and $q_{j}^{f}=q(j h, f \Delta t)$, where $h$ is the mesh size, $\Delta t$ is the time step, $f=0,1,2, \ldots, F$.

The finite difference approximation of equations (1)-(4) using the explicit scheme is as follows

$$
\begin{gathered}
C_{e i}^{f-1} \frac{T_{e i}^{f}-T_{e i}^{f-1}}{\Delta t}=-\frac{q_{e i+1}^{f}-q_{e i-1}^{f}}{2 h}-G_{i}^{f-1}\left(T_{e i}^{f-1}-T_{l i}^{f-1}\right)+Q_{i}^{f-1} \\
C_{l i}^{f-1} \frac{T_{l i}^{f}-T_{l i}^{f-1}}{\Delta t}=-\frac{q_{l i+1}^{f}-q_{l i-1}^{f}}{2 h}+G_{i}^{f-1}\left(T_{e i}^{f-1}-T_{l i}^{f-1}\right)
\end{gathered}
$$

and

$$
\begin{aligned}
& q_{e j}^{f-1}+\tau_{e} \frac{q_{e j}^{f}-q_{e j}^{f-1}}{\Delta t}=-\lambda_{e j}^{f-1} \frac{T_{e j+1}^{f-1}-T_{e j-1}^{f-1}}{2 h} \\
& q_{l j}^{f-1}+\tau_{l} \frac{q_{l j}^{f}-q_{l j}^{f-1}}{\Delta t}=-\lambda_{l j}^{f-1} \frac{T_{l j+1}^{f-1}-T_{l j-1}^{f-1}}{2 h}
\end{aligned}
$$


After the mathematical manipulations one obtains [2]

$$
\begin{aligned}
& T_{e i}^{f}=\left(1-\frac{(\Delta t)^{2} \lambda_{e i-1}^{f-1}}{4 h^{2} \tau_{e} C_{e i}^{f-1}}-\frac{(\Delta t)^{2} \lambda_{e i+1}^{f-1}}{4 h^{2} \tau_{e} C_{e i}^{f-1}}-\frac{G_{i}^{f-1} \Delta t}{C_{e i}^{f-1}}\right) T_{e i}^{f-1}+\frac{(\Delta t)^{2} \lambda_{e i-1}^{f-1}}{4 h^{2} \tau_{e} C_{e i}^{f-1}} T_{e i-2}^{f-1}+ \\
& \frac{(\Delta t)^{2} \lambda_{e i+1}^{f-1}}{4 h^{2} \tau_{e} C_{e i}^{f-1}} T_{e i+2}^{f-1}+\frac{G_{i}^{f-1} \Delta t}{C_{e i}^{f-1}} T_{l i}^{f-1}+\frac{\Delta t\left(\tau_{e}-\Delta t\right)}{2 h \tau_{e} C_{e i}^{f-1}}\left(q_{e i-1}^{f-1}-q_{e i+1}^{f-1}\right)+\frac{Q_{i}^{f-1} \Delta t}{C_{e i}^{f-1}} \\
& T_{l i}^{f}=\left(1-\frac{(\Delta t)^{2} \lambda_{l i-1}^{f-1}}{4 h^{2} \tau_{l} C_{l}}-\frac{(\Delta t)^{2} \lambda_{l i+1}^{f-1}}{4 h^{2} \tau_{l} C_{l}}-\frac{G_{i}^{f-1} \Delta t}{C_{l}}\right) T_{l i}^{f-1}+\frac{(\Delta t)^{2} \lambda_{l i-1}^{f-1}}{4 h^{2} \tau_{l} C_{l}} T_{l i-2}^{f-1}+ \\
& \frac{(\Delta t)^{2} \lambda_{l i+1}^{f-1}}{4 h^{2} \tau_{l} C_{l}} T_{l i+2}^{f-1}+\frac{G_{i}^{f-1} \Delta t}{C_{l}} T_{e i}^{f-1}+\frac{\Delta t\left(\tau_{l}-\Delta t\right)}{2 h \tau_{l} C_{l}}\left(q_{l i-1}^{f-1}-q_{l i+1}^{f-1}\right)
\end{aligned}
$$

and

$$
\begin{gathered}
q_{e j}^{f}=\frac{\tau_{e}-\Delta t}{\tau_{e}} q_{e j}^{f-1}-\frac{\lambda_{e j}^{f-1} \Delta t}{2 h \tau_{e}}\left(T_{e j+1}^{f-1}-T_{e j-1}^{f-1}\right) \\
q_{l j}^{f}=\frac{\tau_{l}-\Delta t}{\tau_{l}} q_{l j}^{f-1}-\frac{\lambda_{l j}^{f-1} \Delta t}{2 h \tau_{l}}\left(T_{l j+1}^{f-1}-T_{l j-1}^{f-1}\right)
\end{gathered}
$$

where

$$
\begin{aligned}
& \lambda_{e i-1}^{f-1}=\frac{\lambda_{e i}^{f-1}+\lambda_{e i-2}^{f-1}}{2}, \quad \lambda_{e i+1}^{f-1}=\frac{\lambda_{e i}^{f-1}+\lambda_{e i+2}^{f-1}}{2} \\
& \lambda_{l i-1}^{f-1}=\frac{\lambda_{l i}^{f-1}+\lambda_{l i-2}^{f-1}}{2}, \quad \lambda_{l i+1}^{f-1}=\frac{\lambda_{l i}^{f-1}+\lambda_{l i+2}^{f-1}}{2} \\
& \lambda_{e j}^{f-1}=\frac{\lambda_{e j-1}^{f-1}+\lambda_{e j+1}^{f-1}}{2}, \quad \lambda_{l j}^{f-1}=\frac{\lambda_{l j-1}^{f-1}+\lambda_{l j+1}^{f-1}}{2}
\end{aligned}
$$

It should be pointed out that adequate stability criteria for the explicit scheme must be fulfilled [2]. 


\section{Results of computations}

The gold film of thickness $L=100 \mathrm{~nm}\left(1 \mathrm{~nm}=10^{-9} \mathrm{~m}\right)$ is considered. The initial temperature is equal to $T_{p}=300 \mathrm{~K}$. The constants in equations (13), (14) are the following: $\chi=353 \mathrm{~W} /(\mathrm{mK}), \eta=0.16, A_{e}=1.2 \cdot 10^{7} 1 /\left(\mathrm{K}^{2} \mathrm{~s}\right), B_{l}=1.23 \cdot 10^{11} 1 /(\mathrm{Ks})$ and $G_{r t}=2.2 \cdot 10^{16} \mathrm{~W} /\left(\mathrm{m}^{3} \mathrm{~K}\right)$ [9]. The Fermi temperature is equal to $T_{F}=64200 \mathrm{~K}$ and the density of electrons is equal to $N=5.9 \cdot 10^{28} 1 / \mathrm{m}$ (cf. equation (11)) [9]. The other parameters are as follows: thermal conductivity of lattice $\lambda_{l}=315 \mathrm{~W} /(\mathrm{mK})$, volumetric specific heat of lattice $C_{l}=2.5 \mathrm{MJ} /\left(\mathrm{m}^{3} \mathrm{~K}\right)$, electrons relaxation time $\tau_{e}=0.04 \mathrm{ps}$, phonons relaxation time $\tau_{l}=0.8 \mathrm{ps}$ [1], reflectivity $R=0.93$, optical penetration depth $\delta=15.3 \mathrm{~nm}$. The problem is solved using the finite difference method under the assumption that $\Delta t=0.002 \mathrm{ps}$ and $h=1 \mathrm{~nm}$.

In Figure 3 the electrons and lattice temperature at the irradiated surface $x=0$ for the laser intensity $I_{0}=4182 \mathrm{~W} / \mathrm{m}^{2}$ and the characteristic time of laser pulse $t_{p}=100 \mathrm{ps}$ is shown.

It should be pointed out that the results are compared with those obtained using the dependences (8), (9) and the constant value of coupling factor $G=G_{r t}$. The differences between the temperatures for this variant of parameters and those previously assumed are shown in Figures 4 and 5.

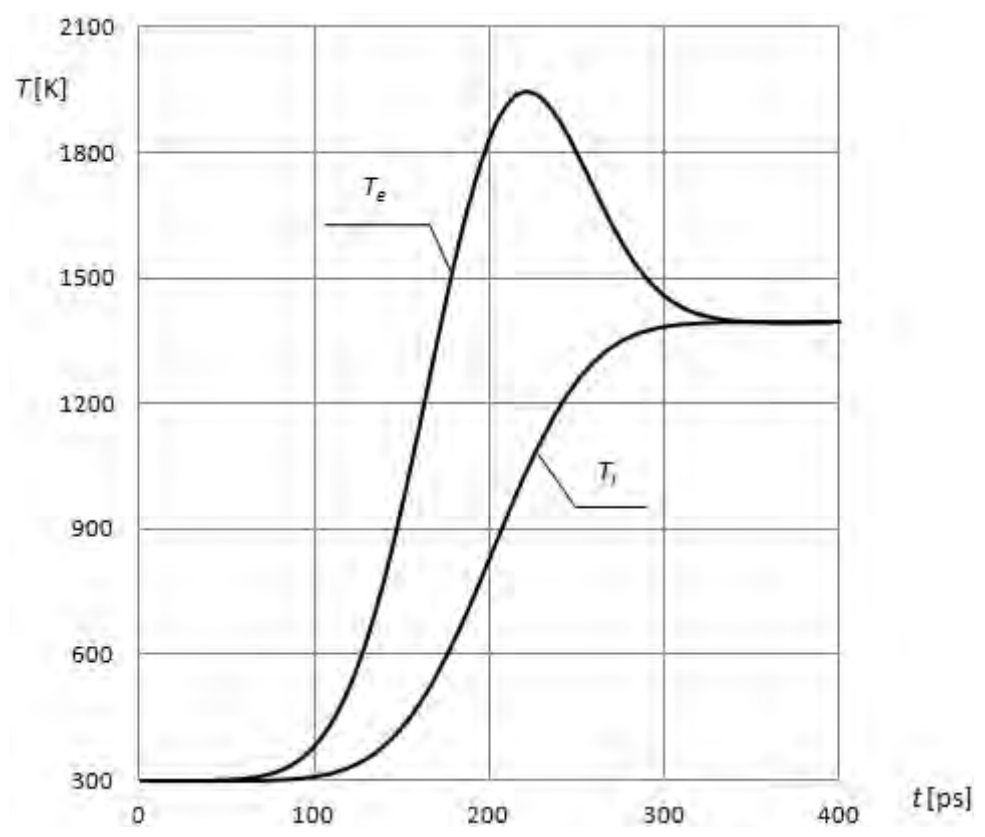

Fig. 3. Electrons and lattice temperature at the irradiated surface 


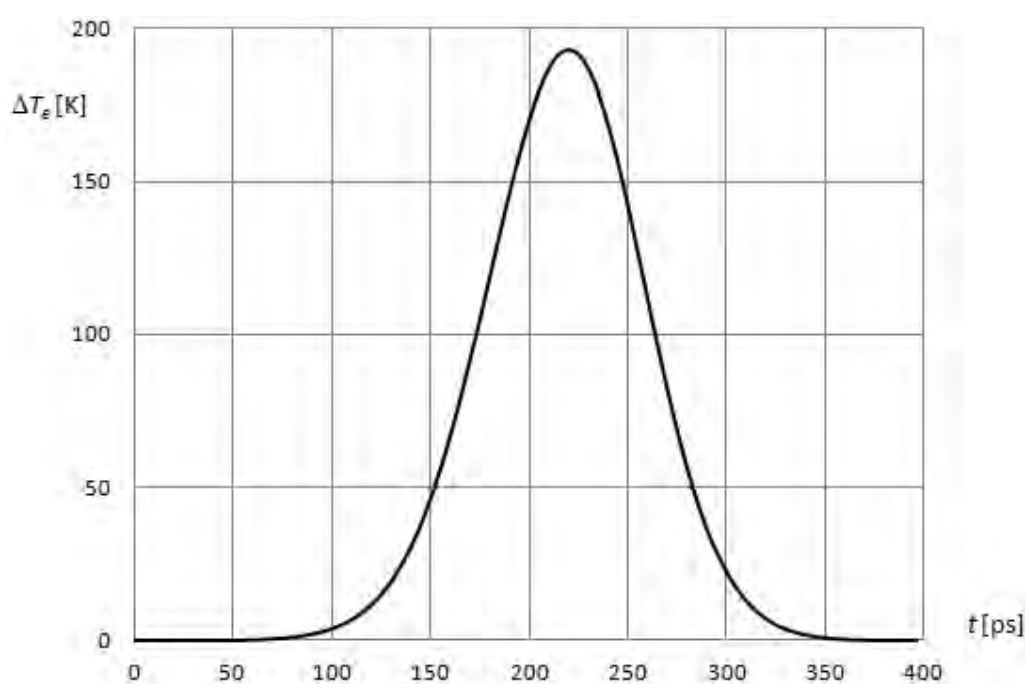

Fig. 4. Electrons temperature differences

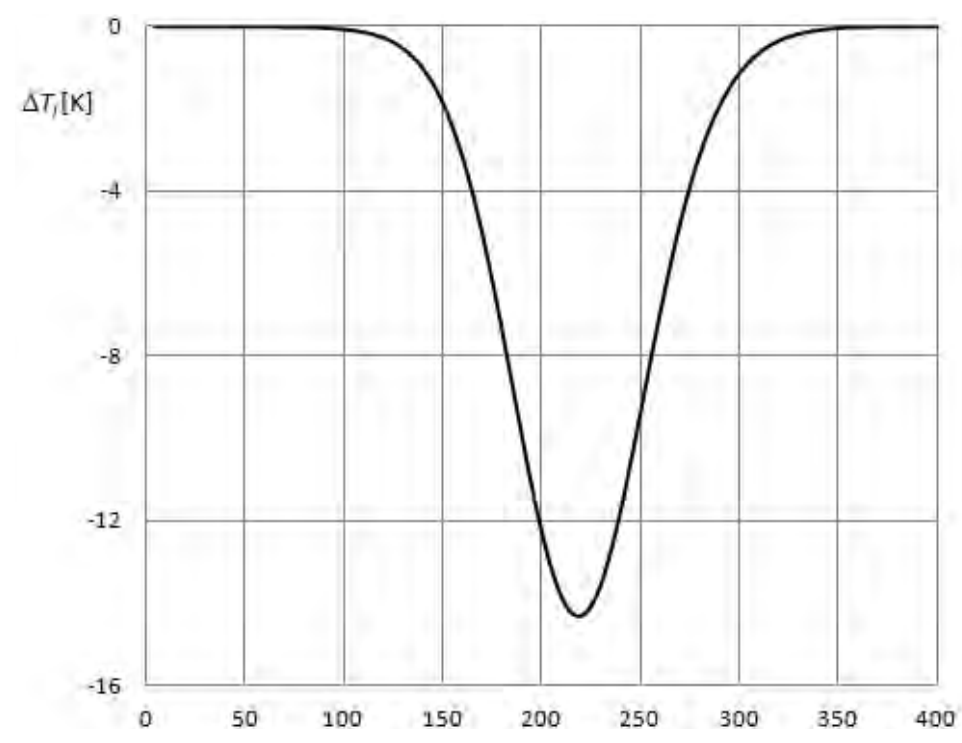

$t[p s]$

Fig. 5. Lattice temperature differences

\section{Conclusions}

The problem of the heat exchange proceeding in the domain of thin metal film subjected to the ultrashort laser pulse described by the two-temperature model is considered. Two sets of parameters occurring in the model have been taken into account and the differences in the electrons and lattice temperatures between them are presented. Temperature-dependent parameters and the way they are estimated 
have more influence on the electrons temperature distribution than on the lattice temperature distribution, but in the case considered the differences are not big.

\section{Acknowledgement}

This work is supported by the project No. 2012/05/B/ST8/01477 and sponsored by the Polish National Science Centre.

\section{References}

[1] Chen J.K., Beraun J.E., Numerical study of ultrashort laser pulse interactions with metal films, Numerical Heat Transfer, Part A 2001, 40, 1-20.

[2] Dziatkiewicz J., Kuś W., Majchrzak E., Burczyński T., Turchan Ł., Bioinspired identification of parameters in microscale heat transfer, International Journal for Multiscale Computational Engineering 2014, 12(1), 79-89.

[3] Dziatkiewicz J., Analiza przepływu ciepła w mikroobszarach z wykorzystaniem modeli dwutemperaturowych, Politechnika Śląska, Gliwice 2012.

[4] Majchrzak E., Dziatkiewicz J., Identification of electron-phonon coupling factor in a thin metal film subjected to an ultrashort laser pulse, Computer Assisted Methods in Engineering and Science 2012, 19, 383-392.

[5] Smith A.N., Norris P.M., Microscale heat transfer, Chapter 18 [in:] Heat Transfer Handbook, John Wiley \& Sons, 2003.

[6] Lin Z., Zhigilei L.V., Electron-phonon coupling and electron heat capacity of metals under conditions of strong electron-phonon nonequilibrium, Physical Review B 2008, 77, 075133-1075133-17.

[7] Majchrzak E., Poteralska J., Two-temperature microscale heat transfer model, Part I: Determination of electron parameters, Scientific Research of the Institute of Mathematics and Computer Science 2010, 1(9), 99-108.

[8] Majchrzak E., Poteralska J., Two-temperature microscale heat transfer model. Part II: Determination of lattice parameters, Scientific Research of the Institute of Mathematics and Computer Science 2010, 1(9), 109-120.

[9] Chen J.K., Tzou D.Y., Beraun J.E., A semiclassical two-temperature model for ultrafast laser heating, International Journal of Heat and Mass Transfer 2006, 49, 307-316.

[10] Huang J., Baheti K., Chen J.K., Zhang Y., An axisymmetric model for solid-liquid-vapor phase change in thin metal films induced by an ultrashort laser pulse, Frontiers in Heat and Mass Transfer 2011, 2, 013005. 